\title{
A Low-Cost, Flexible Pressure Capacitor Sensor Using Polyurethane for Wireless Vehicle Detection
}

\author{
Chien Khong Duc ${ }^{1,2} \mathbb{C}^{\text {, Van-Phuc Hoang }}{ }^{1}{ }^{(\mathbb{D}}$, Duy Tien Nguyen $^{3}$ and Toan Thanh Dao ${ }^{2, *}$ \\ 1 Faculty of Radio-Electronic Engineering, Le Quy Don Technical University, No. 236 Hoang Quoc Viet Street, \\ Co Nhue 1 Ward, Bac Tu Liem District, Hanoi 100000, Vietnam \\ 2 Faculty of Electrical-Electronic Engineering, University of Transport and Communications, No. 3 Cau Giay \\ Street, Lang Thuong Ward, Dong Da District, Hanoi 100000, Vietnam \\ 3 Faculty of Civil Engineering, University of Transport and Communications, No. 3, Cau Giay Street, \\ Lang Thuong Ward, Dong Da District, Hanoi 100000, Vietnam \\ * Correspondence: daotoan@utc.edu.vn; Tel.: +84-979-379-099
}

Received: 19 June 2019; Accepted: 25 July 2019; Published: 27 July 2019

\begin{abstract}
Detection of vehicles on the road can contribute to the establishment of an intelligent transportation management system to allow smooth transportation and the reduction of road accidents. Thus far, an efficient and low-cost polymer flexible pressure sensor for vehicle detection is lacking. This paper presents a flexible sensor for vehicle sensing and demonstrates a wireless system for monitoring vehicles on the road. A vehicle sensor was fabricated by sandwiching a polyurethane material between aluminum top/bottom electrodes. The sensing mechanism was based on changes in capacitance due to variation in the distance between the two electrodes at an applied external pressure. A clear response against a pressure load of $0.65 \mathrm{Mpa}$ was observed, which is the same pressure as that of the car tire area in contact with the road. Significantly, the sensor was easy to embed on the road line due to its mechanical flexibility and large size. A field test was carried out by embedding the sensor on the road and crossing the sensor with a car. Moreover, the signal displayed on the tablet indicated that the sensing system can be used for wireless detection of the axle, speed, or weight of the vehicle on the road. The findings suggest that the flexible pressure sensor is a promising tool for use as a low-cost vehicle detector in future intelligent transportation management.
\end{abstract}

Keywords: flexible pressure sensor; polyurethane polymer; wireless data acquisition (DAQ); non-intrusive monitoring; vehicle detection

\section{Introduction}

Vehicle detection is an important process in intelligent transport management systems, as it allows the collection of big data on vehicles' speeds and weights as well as the traffic intensity, which helps to enhance smooth transportation and to reduce road accidents [1]. Non-intrusive and intrusive sensor technologies are often employed for monitoring [1-11]. A laser sensor, a temperature sensor, or an image-based sensor have been used for vehicle detection that is based on a change in laser light intensity, temperature value, or imaging property due to the vehicle appearance [6-8]. These approaches have high potential to estimate traffic volume and vehicle classification. However, they are negatively affected by the environmental factors, for example fog, snow, rain, or shadow and the installation and maintenance of such sensor systems result in a relative high cost [8]. Recently, induction loops are most commonly used due to their low-cost and high reliability. The operating concept is that when a vehicle passes over the loop, it induces a current change in the wire loop [1-3]. The limitation of such sensors is that the installation process leads to significant damage to an extensive area of road surface. A recent study employed anisotropic magnetoresistance sensors [9-11]. Determination of 
vehicles was realized because the metal construction of a vehicle distorts the magnetic field of the Earth. However, the drawback of this sensing method is that it has a relatively high cost, and is likely to contain noise signals due to interference from neighboring vehicles on the road or metal materials from other sources.

On the other hand, in recent years, a pressure sensor using polymer materials has received much attention among academics and industry due to its unique advantages, including low-temperature processing, low manufacturing costs, mechanical flexibility, and the potential to work in a large area $[7,8,12-23]$. These characteristics indicate the potential to construct the next generation of IoT (Internet of Things) sensing nodes, where the sensor is required to not only be low-power and low-cost, but also, be compatible with many surface types [11,12]. Structurally, the sensor contains an active polymer material layer sandwiched between two electrodes [8,12-23]. Based on the physical phenomena of piezoresistivity, piezocapacity, and piezoelectricity, when the applied pressure changes, the resistance, capacitance, or electricity of the sandwiched layer varies, which, in turn, causes the output electrical signal of the device to change. In general, those sandwiched layers are mostly obtained through the use of sensing material system such as a poly(vinylidenefluoride-co-trifluoroethylene (P(VDF-TrFE)), a polydimethylsiloxane (PDMS) or a mixture of polymer and nanoparticles or polymers doped with carbon nanotube [8,12-23]. Based on its advantages, the flexible pressure sensor is expected to be used in vehicle detection without damaging the transportation infrastructure, as it can be adhered to the road. In addition, the sensor may not be affected by the magnetic field of Earth like an anisotropic magnetoresistance device since the polymer is a non-metallic material. In particular, literature studies have mainly focused on the use of flexible sensors with a pressure from a low-regime $(0-0.01 \mathrm{Mpa})$ to medium-regime (0.01-0.1 Mpa) for human health monitoring, such as heart rate monitors, EEG signals, object tracing, collision events, or weight measurements [11-20]. In addition, a sensor with a higher measurement range has recently attracted a great deal of attention because of its promising applications in smart transportation [7,8,24]. For example, Ding et al. demonstrated a sensor device by adding spiky spherical nickel particles into liquid silicon rubber. In such nickel particles-based sensor, the sensed value was in the range of 0-6.4 Mpa [7]. Mohiuddin and Van Ho fabricated a pressure sensor by mixing the multiwalled carbon nanotubes in a polyether ether ketone polymer matrix, which resulted in the measurement value up to $40 \mathrm{Mpa}$ [24]. Overall, those sensors are capable for utilization in vehicle sensing because their measurement ranges are well overlapped the pressure of car tires on the road (0.4-0.7 Mpa, Ref. [25]). However, the device fabrication technique including the control of uniform dispersion of dopants in the polymer matrix is relatively complicated and costly, which limits the wider use of sensors to practical public transportation.

In this work, we demonstrate a vehicle detection capacitor sensor fabricated with a polyurethane material and a thermal lamination technique. We find that by applying an external force to the sensor, the thickness of the polyurethane dielectric layer is decreased, resulting in increased capacitance of the sensor. The measurement value of the sensor is up to $0.65 \mathrm{Mpa}$, which matches the pressure of a vehicle tire well $[25,26]$. The best sensitivity of the sensor is obtained to be $8 \times 10^{-2} \mathrm{kPa}^{-1}$. The sensor is easy to mount on a road without re-construction of the road surface due to its flexibility. Additionally, wireless data acquisition (DAQ) together with a tablet computer-based application (App) is also developed for vehicle detection. Real-time vehicle sensing is successfully achieved by the sensor with the wireless DAQ system embedded on the road and the tablet computer.

\section{Experimental Method}

Figure 1 shows a graphical illustration of the flexible sensor where a capacitor structure was utilized because of its simple structure, good stability, and low power consumption [3,9-12]. For sensor fabrication, aluminum (Al) foil electrodes purchased from Sigma-Aldrich (resistivity of $2.6548 \mu \Omega \mathrm{cm}$; thickness of $30 \mu \mathrm{m}$, Singapore) were initially sputter-etched to remove potential contamination or native oxide structures. In short, the $\mathrm{Al}$ foil was loaded in the etching chamber, under a vacuum background of $10^{-6}$ Torr. Subsequently, argon gas with a flow rate of $20 \mathrm{sccm}$ is inlet into the chamber, and the 
etching pressure is controlled to be $0.1 \mathrm{~Pa}$. An automatic tuning process is applied to generate plasma atmosphere, and to start an etching process. The etching power is set to be $30 \mathrm{~W}$, corresponding to the etching rate of $0.5 \mathrm{~nm} / \mathrm{min}$. For our case, we performed this cleaning process about $5-10 \mathrm{~min}$ to ensure a perfect conductive surface of $\mathrm{Al}$ foil. A $100-\mu \mathrm{m}$-thick polymer film of polyurethane (Tensile strength $=38 \mathrm{Mpa}$, Sanyo Chemical, Tokyo, Japan) was employed as an active layer, which was sandwiched between two aluminum electrodes, followed by lamination at $80{ }^{\circ} \mathrm{C}$ twice (Figure 2a). The sensor size was determined by the overlapping area of the two electrodes to be $70 \mathrm{~mm} \times 70 \mathrm{~mm}$. This large size was used with the aim of being suitable for an application in car tire tracking. Subsequently, a copper wire was attached to the electrode to form the connecting lead of the sensor. Finally, for protection, the sensor was also covered with a plastic film by the lamination method at $80^{\circ} \mathrm{C}$ (Figure $2 \mathrm{~b}$ ). The rollers of the laminator in here are to help to flatten sandwiched polymer and increase the adhesive functionality. Figure 2c shows photos of the fabricated sensor in a (left) normal or (right) flexible mode. To examine the homogeneity of sensor layer after lamination, a cross-sectional image of the sensor was taken with a microscope. As can be seen in Figure 3, each layer is well realized, indicating a homogenous property of device layer.

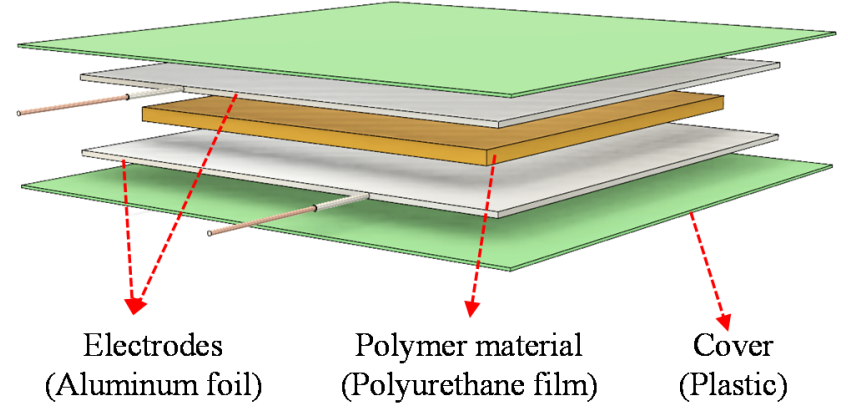

Figure 1. Structure of the flexible pressure.

(a)

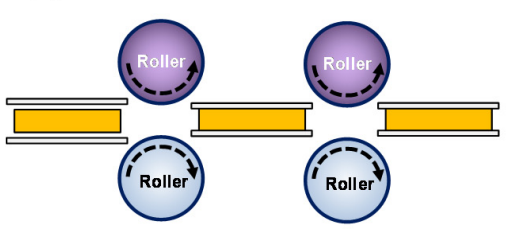

(b)

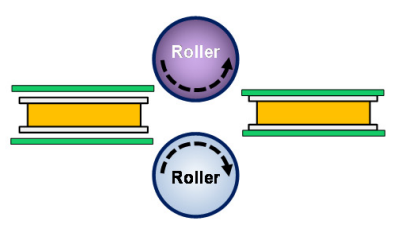

(c)

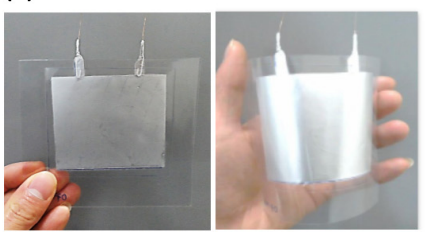

Figure 2. Illustration of the sensor fabrication process: (a) electrode layer formation, (b) encapsulation, (c) photos of the sensor in (left) normal or (right) flexible mode.

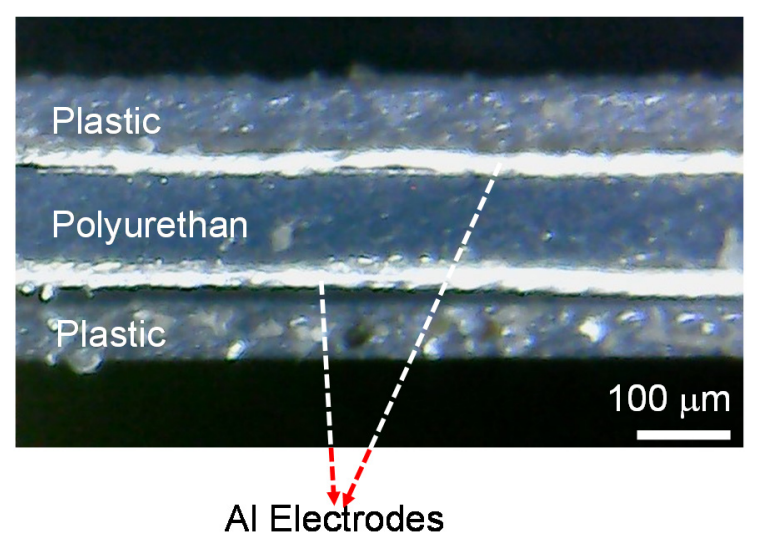

Figure 3. Cross-sectional image of fabricated sensor. 
Characterization of the sensors was performed using a universal compression testing machine (UH 500-kN, SHIMADZU, Kyoto, Japan) and a capacitance meter (YF-150, Tenmars Electronics, Taipei, Taiwan) at a frequency of $100 \mathrm{~Hz}$ at room temperature. The dynamic range of the sensor was tuned to vary from 0 to around $0.65 \mathrm{Mpa}$, which is the same pressure as the car tire area contacts with the road. To examine the influence of the polymer thickness in sensing, devices with different film thicknesses of polyurethane $(200,300$, and $500 \mu \mathrm{m})$ were also fabricated using the same experimental method.

\section{Results and Discussion}

\subsection{Sensor Characteristics}

Figure 4 shows the capacitance-pressure characteristics of the sensors when the pressure varies from 0 to $0.65 \mathrm{Mpa}$. The initial capacitance values were measured to be $0.13,0.19,0.083$, and $0.050 \mathrm{pF} / \mathrm{mm}^{2}$ from the devices with polyurethane thicknesses of 100, 200, 300, and $500 \mu \mathrm{m}$, respectively. A steep increment in capacitance was observed at the low-pressure region and the increment tended to saturate with an increasing applied pressure $(p)$. A similar characteristic is also typical among capacitive sensor systems [17,19-21], suggesting that our device sensors were well fabricated.
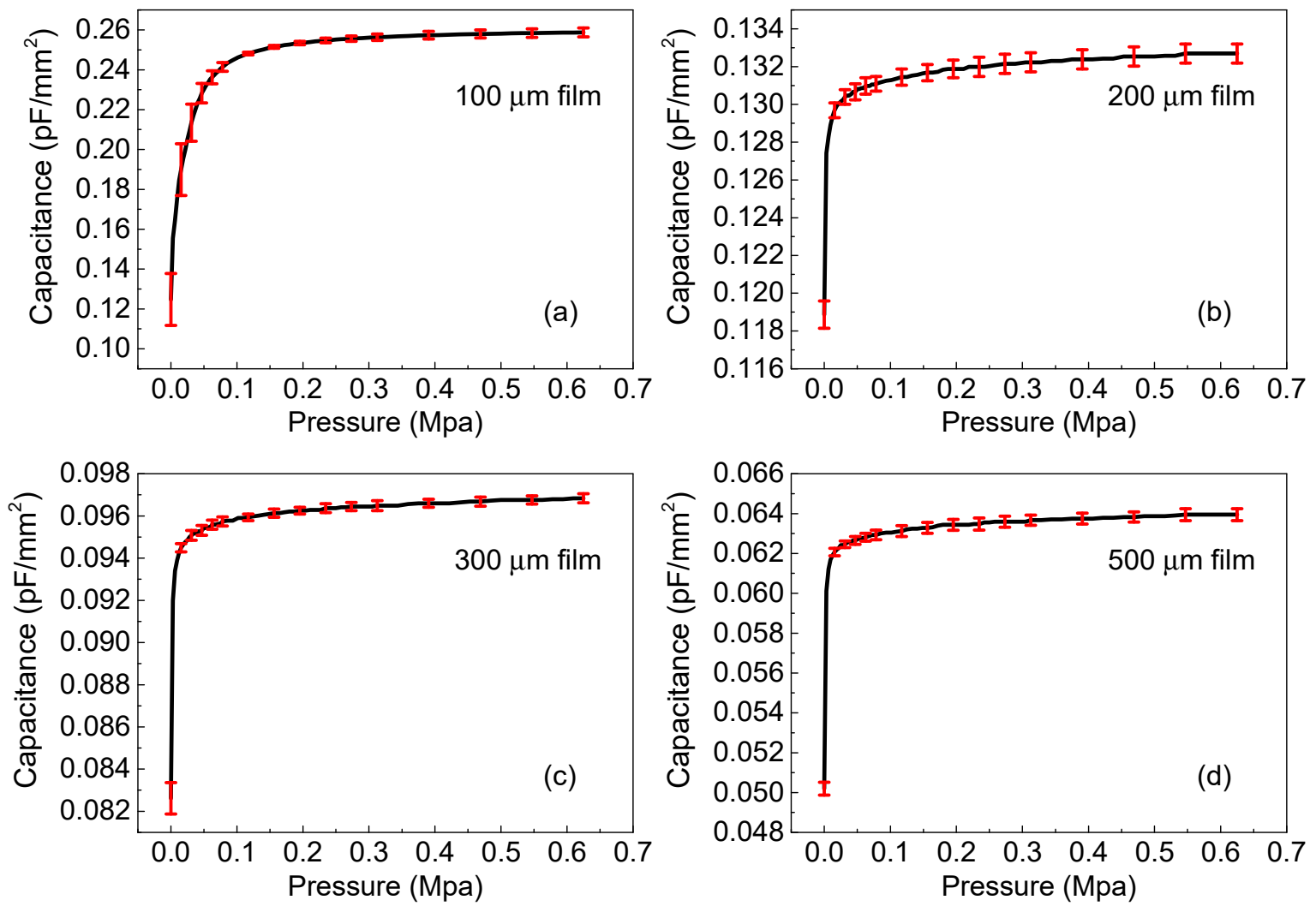

Figure 4. Capacitance-pressure characteristics of sensors with thicknesses of (a) 100, (b) 200, (c) 300, and (d) $500 \mu \mathrm{m}$.

To understand the operating mechanism of the fabricated sensors, we carried out further analysis, as follows. Fundamentally, the capacitance of two parallel electrodes can be described by the below equation:

$$
C=\varepsilon \frac{A}{t}
$$

where $\varepsilon, A$, and $t$ are the dielectric constant, the area of overlap of the two electrodes, and the thickness of the dielectric layer (or the distance between the electrodes), respectively. As shown by Equation (1), under application of pressure, the change in $C$ can be considered for the following reasons: (1) a change 
in the dielectric constant of the dielectric layer, (2) a change in the device area, or (3) a change in the distance between two electrodes. However, mechanism (1) can be neglected because the dielectric constant of the polymer material obtained during the sensor under compression was almost unchanged to be about 2.2. If the device area change is the reason, the capacitance has difficulty rapidly increasing like in curve Figure 4 . Thus, we assume that the decrease in the thickness thanks to the elasticity of the polymer is attributed to the operating mechanism. Based on that consideration, the proposed operation mechanism of the sensor is presented in Figure 5. Under application of force to the sensor electrode, the thickness of the film decreases, which, in turn, results in the capacitance increasing in accordance with Equation (1). In contrast, the applied force is removed from the interface; consequently, the capacitance reverts to the initial value.

(a)

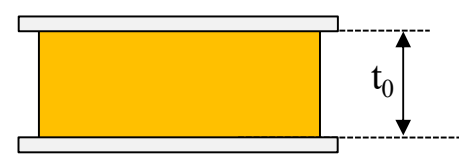

(b)

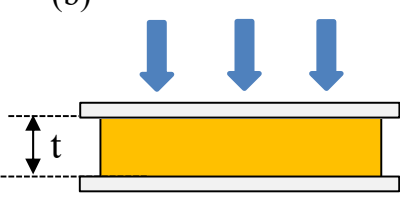

Figure 5. Illustration of the operating mechanism in pressure capacitor sensor: (a) without pressure and (b) under pressure.

We conducted an estimation to investigate the variation in $t$ with external pressure loading. From Equation (1), $t$ was calculated using the following equation: $t=\varepsilon A / C$. For example, in Figure 6, the capacitance change and the thickness change with respect to the external pressure are plotted for the $100 \mu \mathrm{m}$ thick film sensor. The thickness $t$ of the layer tends to decrease with increasing pressure which is the opposite tendency to the chance in capacitance.

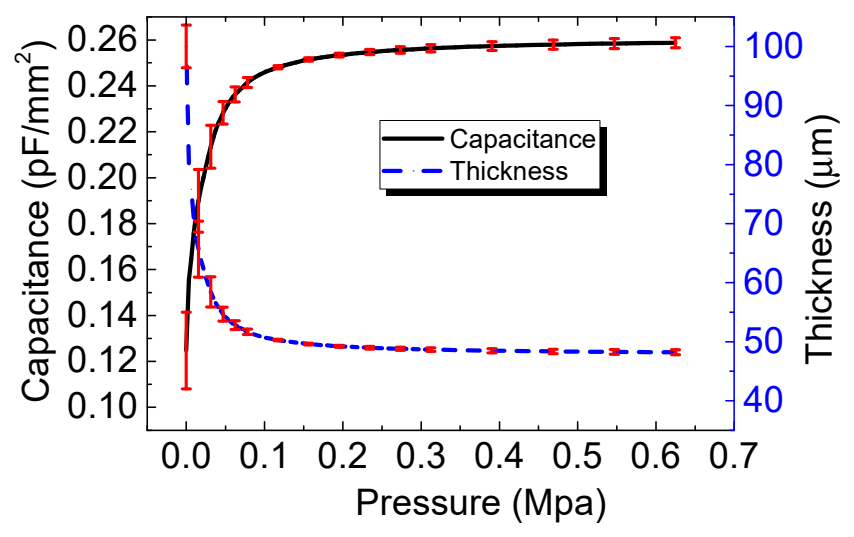

Figure 6. Capacitance and the thickness change with respect to the external pressure.

In sensors for human health monitoring [16-23], the void structures have widely used to enhance sensitivity of the sensors, but those also result in the measurement value of below $0.1 \mathrm{Mpa}$. In our study, thanks to the utilization of a polyurethane film with a tensile strength of $38 \mathrm{Mpa}$, the ability of the sensor to measure pressure values of up to $0.65 \mathrm{Mpa}$.

On the other hand, in order to quantify the influence of the active thickness, the effective capacitance change was estimated with the following equation [21]:

$$
\frac{\Delta C}{C_{0}}=\frac{C_{\max }-C_{0}}{C_{0}}
$$

where $C_{\max }$ is the capacitance at $p=0.65 \mathrm{Mpa}$, and $C_{0}$ is the capacitance at $p=0$. We found that the $\Delta C / C_{0}$ significantly decreased from 1.070 to $0.274,0.172$ and 0.116 when the polyurethane thickness 
increased from 100 to 200,300 and $500 \mu \mathrm{m}$, respectively. This could be due to the fact that the reduction in the distance between the top and bottom electrodes in a thin device is more considerable.

The sensitivity $(S)$ was calculated using the equation [21]:

$$
S=\frac{\delta\left(\Delta C / C_{0}\right)}{\delta P}
$$

The $S$ at three pressure ranges and different film thicknesses is shown in Table 1 . The thinner device exhibits a higher $S$, which results from its factor of $\Delta C / C_{0}$ being higher. The $S$ values in the range of $0.2-0.65 \mathrm{Mpa}$ for each device are $1.18 \times 10^{-4}, 5.57 \times 10^{-5}, 5.07 \times 10^{-5}$, and $8.35 \times 10^{-5}$, respectively. Overall, the $100 \mu \mathrm{m}$-based sensor exhibits the best performance in terms of $\Delta C / C_{0}$ and S. Significantly, an $S$ value of up to $8 \times 10^{-2} \mathrm{kPa}^{-1}$ was obtained from the $100 \mu \mathrm{m}$ based-sensor at $0.003 \mathrm{Mpa}$, which is comparable to the previous pressure sensor systems [19-21].

Table 1. Sensitivity at different pressure ranges or film thicknesses.

\begin{tabular}{ccccc}
\hline \multirow{2}{*}{ Pressure Range (Mpa) } & \multicolumn{4}{c}{ Sensitivity $\left(\mathbf{k P a}^{-\mathbf{1}}\right)$} \\
\cline { 2 - 5 } & $\mathbf{t}=\mathbf{1 0 0} \boldsymbol{\mu \mathbf { m }}$ & $\mathbf{t}=\mathbf{2 0 0} \boldsymbol{\mu \mathbf { m }}$ & $\mathbf{t}=\mathbf{3 0 0} \boldsymbol{\mu \mathbf { m }}$ & $\mathbf{t = 5 0 0} \boldsymbol{\mu m}$ \\
\hline $0-0.03$ & $2.42 \times 10^{-2}$ & $3.40 \times 10^{-3}$ & $5.33 \times 10^{-3}$ & $8.77 \times 10^{-3}$ \\
\hline $0.03-0.2$ & $2.98 \times 10^{-3}$ & $1.64 \times 10^{-4}$ & $1.89 \times 10^{-4}$ & $2.30 \times 10^{-4}$ \\
\hline $0.20-0.65$ & $1.18 \times 10^{-4}$ & $5.57 \times 10^{-5}$ & $5.07 \times 10^{-5}$ & $8.35 \times 10^{-5}$ \\
\hline
\end{tabular}

In order to confirm the reproducibility of the change in capacitance values under constant $p$, the repeatable properties of the sensor in response to a constant $p$ of $0.65 \mathrm{Mpa}$ were checked with the $100 \mu \mathrm{m}$ based-sensor (Figure 7). In the first cycle, capacitance at $p=0 \mathrm{Mpa}$ was not returned to the initial value when the pressure was released. This would be due to the deformation of the polyurethane film. However, in the second cycle, the repeatable characteristics were well observed as shown in Figure 7a. Moreover, a pressure-sensing characteristics under various values of $p$ were investigated, wherein the $p$ values of $0.08,0.2,0.65,1.0$, and $1.5 \mathrm{Mpa}$ were applied. As can be seen in Figure $7 \mathrm{~b}$, the sensor presents stable responses with a great repeatability. Overall, the sensors can be considered durable for the pressure and available for vehicle sensing.
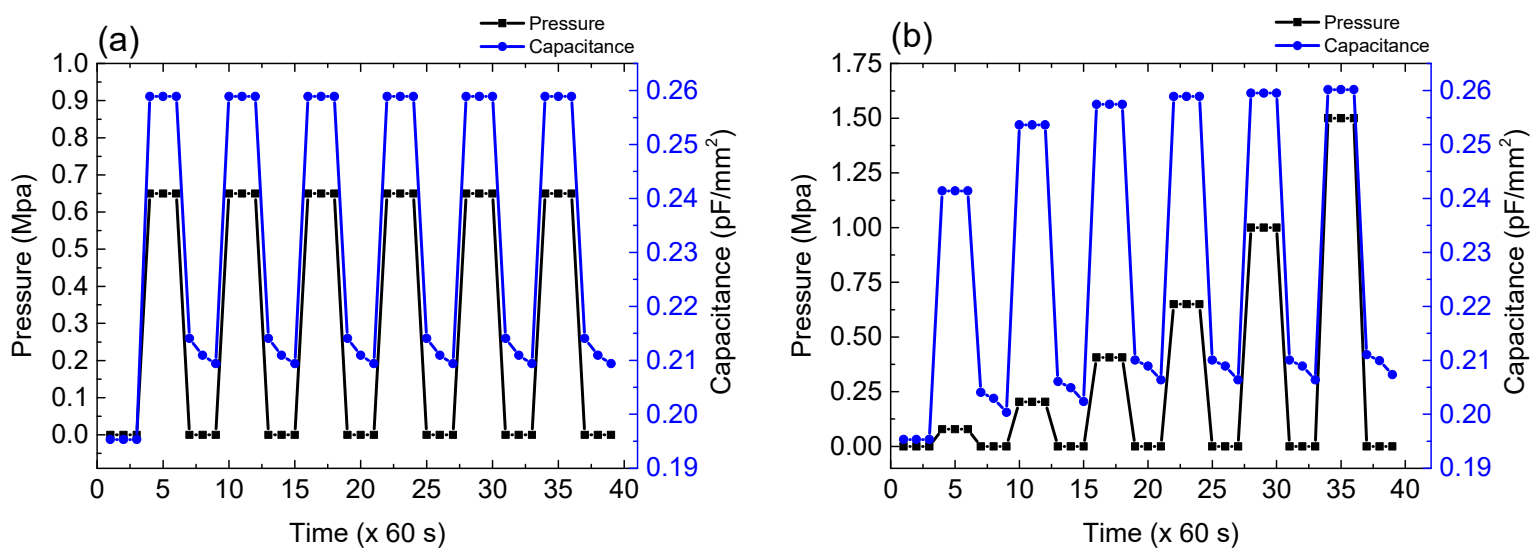

Figure 7. Endurance cycle test of the $100 \mu \mathrm{m}$-based sensor in response to (a) constant $p$ and (b) various values of $p$.

Figure 8 presents flexibility of polyurethane sensor where the capacitance of the sensor was measured at different bent radii of curvature ranging from $200 \mathrm{~mm}$ to infinite (normal state). As can be seen in Figure 8, a relative steep increment in capacitance was observed at the bent radius from 200 to $500 \mathrm{~mm}$ and the increment tended to saturate at bent radius above $500 \mathrm{~mm}$, suggesting that the 
polyurethane sensor can be properly operated at bent radius more than $500 \mathrm{~mm}$. We would like to confirm here that, the ability of flexible operation makes the sensor adapt well to the surface roughness of the asphalt concrete (see Figure S1) which is widely used to construct a road nowadays.

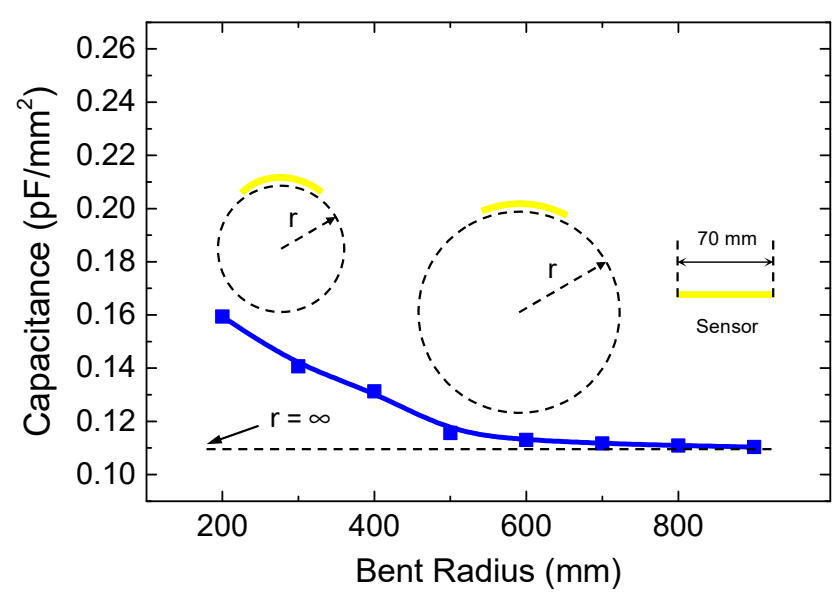

Figure 8. Measured capacitance of capacitor sensor at different bent radius. Inset shows illustration of flexible operation at different bent radius.

\subsection{Development of Wireless $D A Q$}

For the purpose of the field test using the flexible pressure sensor, a completed electronic system using the sensor and a wireless DAQ was designed with the circuit diagram presented in Figure 9, where a NE555 based-oscillator $[27,28]$ was utilized to convert the sensing capacitance to a pulse train and then digitized with a 10-bit-ADC (analog-to-digital converter). With the aim of constructing a DAQ with a small size and low-power, an IoT microprocessor of L106 32-bit RISC and IEEE $802.11 \mathrm{~b} / \mathrm{g} / \mathrm{n}$ integrated in the ESP8266 module were used. An embedded code for the microprocessor chip was written to process, store, and transmit the sensed data to the tablet computer through a WiFi method. The power supply for the DAQ circuits in DAQ was provided from a 3.7 V-LiPo rechargeable battery. A tablet app written with Android Studio allowed real-time display of the sensed signal and export of the data in Excel format for further analysis. We note that since capacitance might not be returned to the initial value when the pressure was released after the first cycle as shown in Figure 7a, the signal data were normalized before displaying on Tablet.

In order to test durable operation for sensor device system consisting of the flexible sensor, wireless DAQ and Tablet app, a Wheel-Track Device of Hamburg AASHTO T 324-04 with a loaded pressure of 0.6 Mpa was used. As shown in Figure S1 and Video S1a,b, our sensor device system can be well operated for several hundreds of cycles without degradation, suggesting a highly stable sensor device system. 

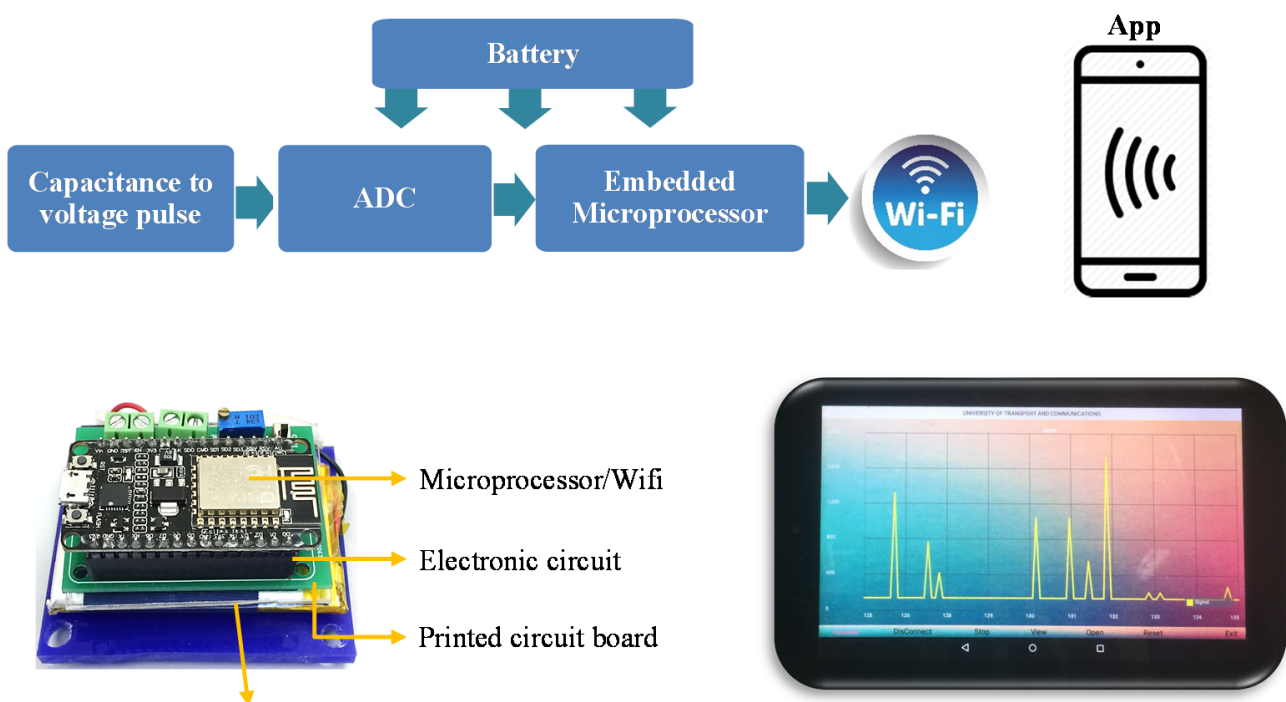

Battery/boost inverter

Figure 9. Diagram of the wireless data acquisition (DAQ) device. Inset shows the printed circuit board (PCB) and tablet with the running app.

\subsection{Demonstration of Vehicle Sensing}

Figure 10 shows a University field test of the vehicle sensing using the system where the sensor was easy to embed between the paper hardcover and was mounted on the road line using tape without any re-construction of the road surface. We would like to note here that our system is advanced compared to current vehicle sensing technology where the road must be re-constructed [1,9]. The sensor is easily mounted on the road due to its flexibility. The relative large sensor size of $70 \mathrm{~mm} \times 70 \mathrm{~mm}$ can make it convenient for matching the footprint of the tire. In this demonstration, a Honda car of 1000-1400 kg with two axles was used as the test vehicle. The signal data for each test were real-time recorded and stored, for example, Video S2a-c show the recorded signal when the car is passing at 0, 5, and $20 \mathrm{~km} / \mathrm{h}$, respectively. Figure 11 shows a typical reconstructed signal of axle load. Based on that, the axle, speed, or weight of the vehicle was deduced.
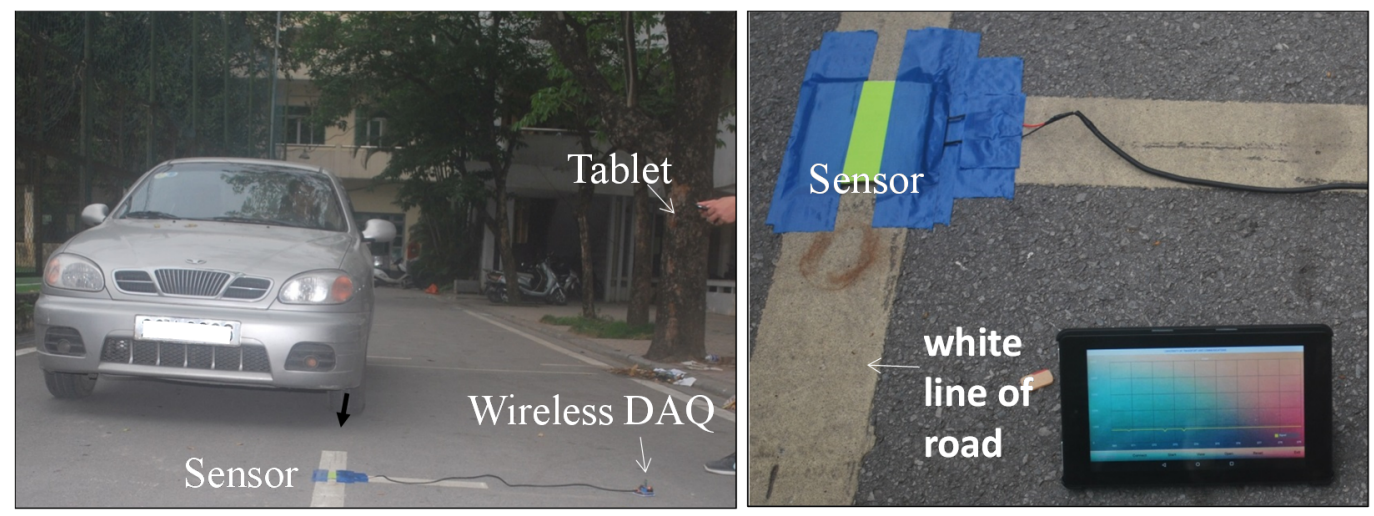

Figure 10. (left) Experimental overview of the vehicle sensing using the system and (right) close-up view of sensor mounted on the road. 


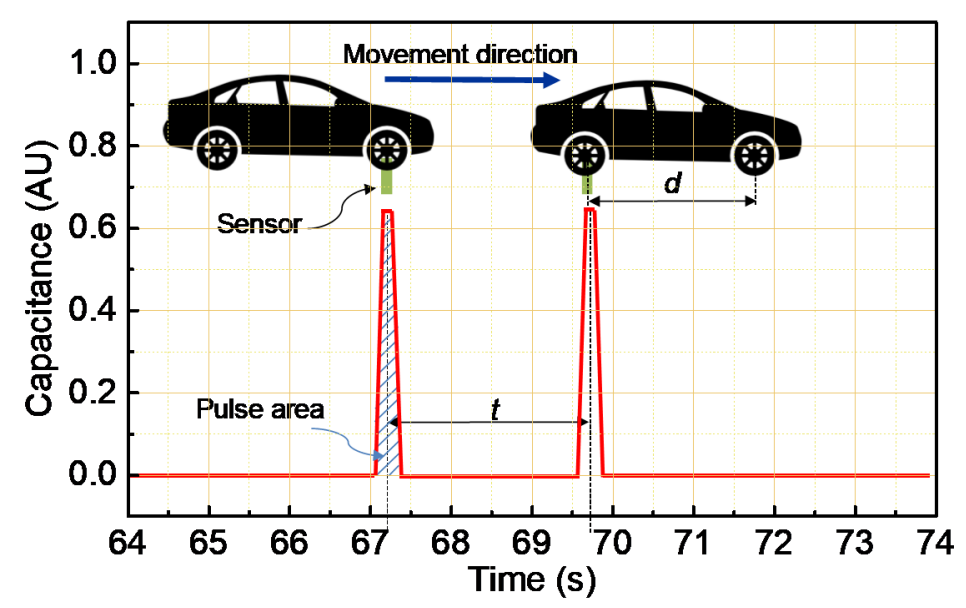

Figure 11. Reconstructed signal of axle load recorded at $5 \mathrm{~km} / \mathrm{h}$. Inset illustrates related parameters to estimate speed or weight vehicle.

The primary goal of the sensor device system is to detect the vehicle axle, leading to a purpose of vehicle classification. Figure 11 shows a typical axle signal reconstructed during a vehicle passing on the sensor (see Video S2b). It is well-known that a peak impulse in signal output corresponds to a vehicle axle $[8,29,30]$. As can be seen, the 2-pulse signal presents accurately a 2-axle car.

The vehicle speed $(V)$ was estimated with the following equation [29]:

$$
V=\frac{d}{t}
$$

where $d$ is the distance between axles of vehicle (Wheelbase) and $t$ is the period between the first and second pulses (Figure 11).

The vehicle weight $(\mathrm{W})$ was estimated with the following equation [29]:

$$
W=V \times A \times K_{W}
$$

where $A$ is the area of pulse (Figure 11) and $K_{W}$ is the calibration factor, which is obtained by using a passing vehicle with known weight and sensor width.

Table 2 lists the true $V$ and calculated $V$ based on sensor output data for a Honda car with a known $d$ of $2600 \mathrm{~mm}$. As shown, the error increases as increasing the true $V$. Overall, the error value is relative high, for example, at true $V$ of $30 \mathrm{Km} / \mathrm{s}$, the error is estimated to be more than $10 \%$. Thus it needs to further study on the algorithm in order to increase the accuracy of the vehicle speed for our sensor system. At the true $V$ of $35 \mathrm{~km} / \mathrm{h}$, a pulse signal cannot be observed. This is because the smaller deformation for the sensor at higher speed, leading to the area of pulse signal becomes narrower as the speed increases. Thus, it can be concluded that the $V$ detection of current sensor device is less than $35 \mathrm{~km} / \mathrm{h}$. On the other aspect, Table 3 shows results of estimated $W$ and errors at $V=5 \mathrm{~km} / \mathrm{h}$ when the true $W$ varied from 1000 to $1400 \mathrm{~kg}$. As shown in Table 3, the calculation of the $W$ is more accurate with an error less than $0.5 \%$. It notes here that the range of true $W$ is narrow because our limitation of weight source facility. However, based on the first results from the estimations of $V$ and $W$, it indicates that the sensor system is capable of determining axle, collecting the data of $V$ or $W$ and is useable for vehicle detection. 
Table 2. Results of estimated $V$ and errors for the different true $V$ values.

\begin{tabular}{cccc}
\hline No. & True V (km/h) & Estimated V (Km/h) & Error (\%) \\
\hline 1 & 2 & 2.02 & 1.00 \\
\hline 2 & 5 & 5.13 & 2.60 \\
\hline 3 & 10 & 10.35 & 3.50 \\
\hline 4 & 15 & 15.61 & 4.06 \\
\hline 5 & 20 & 21.21 & 6.05 \\
\hline 6 & 25 & 26.79 & 7.16 \\
\hline 7 & 30 & 33.25 & 10.83 \\
\hline 8 & 35 & N/A & N/A \\
\hline
\end{tabular}

Table 3. Results of estimated $W$ and errors for the different true $W$ values at $V=5 \mathrm{~km} / \mathrm{h}$.

\begin{tabular}{cccc}
\hline No. & True W $\mathbf{( K g )}$ & Estimated W $\mathbf{( K g )}$ & Error $(\%)$ \\
\hline 1 & 1000 & 999.51 & 0.049 \\
\hline 2 & 1050 & 1048.45 & 0.15 \\
\hline 3 & 1100 & 1104.05 & 0.37 \\
\hline 4 & 1200 & 1205.46 & 0.46 \\
\hline 5 & 1300 & 1296.15 & 0.30 \\
\hline 6 & 1400 & 1404.56 & 0.33 \\
\hline
\end{tabular}

\section{Conclusions}

In conclusion, a flexible sensor for vehicle detection made was fabricated with polyurethane material using a lamination method. The operating mechanism was attributed to the reduction of the thickness of the dielectric layer $t$. Electrical characterization revealed that the $100 \mu \mathrm{m}$-based sensor exhibits the best performance in terms of $\Delta C / C_{0}$ and $S$. The ability of the sensor to measure pressure values of up to $0.65 \mathrm{Mpa}$ shows that it has potential for application in vehicle detection. Moreover, a wireless system based on the fabricated sensor for monitoring vehicles on the road was demonstrated. Owing to its mechanical flexibility and large size, the sensor is easy to embed on the road line. The axle, speed, or weight of the vehicle can be realized based on the app on the tablet computer. The experimental results presented here indicate that the flexible pressure sensor is a promising way to construct a low-cost intelligent transportation management device. However, obviously, the sensor systems have still some problems such as a low-accuracy of the estimated $V$ and low measurement value (less than $35 \mathrm{Km} / \mathrm{h}$ ), suggesting that additional works especially in algorithm need to be performed in order to suppress those limitations. In addition to that, more tested scenarios including utilizations of a wider weight range, real road, or different types of vehicle are necessary to be done to evaluate for further validation of the fabricated sensor device systems.

Supplementary Materials: The following are available online at http://www.mdpi.com/2073-4360/11/8/1247/s1, Figure S1: title, Video S1: title. Figure S1: (a) Sensor mounted on asphalt sample and putting into (b) Sample under test by Wheel-Track Device of Hamburg AASHTO T 324-04. (c) Recorded signal from tablet during device under test. (d) Illustration of testing operation. Video S1a: Overview of durability testing for sensor device with experimental setup. Video S1b: Recorded signal on tablet for durability testing for sensor device with experimental setup. Video S2a: Recorded signal on tablet at $0 \mathrm{~km} / \mathrm{h}$. Video S2b: Recorded signal on tablet at $5 \mathrm{~km} / \mathrm{h}$. Video S2c: Recorded signal on tablet at $20 \mathrm{~km} / \mathrm{h}$.

Author Contributions: T.T.D. conceived the idea; C.K.D. and D.T.N. performed the experiments; C.K.D., V.-P.H., D.T.N. and T.T.D. wrote the paper.

Funding: This work was funded by Vietnam National Foundation for Science and Technology Development (NAFOSTED) under grant number "103.02-2017.34". 
Conflicts of Interest: The authors declare no conflict of interest.

\section{References}

1. Guerrero-Ibanez, J.; Zeadally, S.; Contreras-Castillo, J. Sensor technologies for intelligent transportation systems. Sensors 2018, 18, 1212. [CrossRef] [PubMed]

2. Hu, X.; Yang, L.; Xiong, W. A novel wireless sensor network frame for urban transportation. IEEE Internet Things J. 2015, 2, 586-595. [CrossRef]

3. Jain, N.K.; Saini, R.K.; Mittal, P. A review on traffic monitoring system techniques. Soft Comput. Theor. Appl. 2019, 742, 569-577.

4. Nellore, K.; Hancke, G.P. A survey on urban traffic management system using wireless sensor networks. Sensors 2016, 16, 157. [CrossRef] [PubMed]

5. Tang, Y.; Zhang, C.; Gu, R.; Li, P.; Yang, B. Vehicle detection and recognition for intelligent traffic surveillance system. Multimed. Tools Appl. 2017, 76, 5817-5832. [CrossRef]

6. Klein, L.A. Sensor Technologies and Data Requirements for ITS; Artech House: Boston, MA, USA, 2001.

7. Ding, S.; Han, B.; Dong, X.; Yu, X.; Ni, Y.; Zheng, Q.; Ou, J. Pressure-sensitive behaviors, mechanisms and model of field assisted quantum tunneling composites. Polymer 2017, 113, 105-118. [CrossRef]

8. Han, B.; Zhang, K.; Yu, X.; Kwon, E.; Ou, J. Nickel particle-based self-sensing pavement for vehicle detection. Measurement 2011, 44, 1645-1650. [CrossRef]

9. Markevicius, V.; Navikas, D.; Zilys, M.; Andriukaitis, D.; Valinevicius, A.; Cepenas, M. Dynamic vehicle detection via the use of magnetic field sensors. Sensors 2016, 16, 78. [CrossRef]

10. Daubaras, A.; Markevicius, V.; Navikas, D.; Zilys, M. Analysis of magnetic field disturbance curve for vehicle presence detection. Elektron. Elektrotech. 2014, 5, 80-83. [CrossRef]

11. Daubaras, A.; Zilys, M. Vehicle detection based on magneto-resistive magnetic field sensor. Electron. Electr. Eng. 2012, 118, 27-32. [CrossRef]

12. Liu, Y.; Wang, H.; Zhao, W.; Zhang, M.; Qin, H.; Xie, Y. Flexible, stretchable sensors for wearable health monitoring: Sensing mechanisms, materials, fabrication strategies and features. Sensors 2018, 18, 645. [CrossRef] [PubMed]

13. Zang, Y.; Zhang, F.; Di, C.; Zhu, D. Advances of flexible pressure sensors toward artificial intelligence and health care applications. Mater. Horiz. 2015, 2, 140-156. [CrossRef]

14. Hegde, N.; Bries, M.; Sazonov, E. A comparative review of footwear-based wearable systems. Electronics 2016, 5, 48. [CrossRef]

15. Sekine, T.; Sugano, R.; Tashiro, T.; Sato, J.; Takeda, Y.; Matsui, H.; Kumaki, D.; Domingues Dos Santos, F.; Miyabo, A.; Tokito, S. Fully printed wearable vital sensor for human pulse rate monitoring using ferroelectric polymer. Sci. Rep. 2018, 8, 4442. [CrossRef] [PubMed]

16. Laflamme, S.; Ubertini, F.; Saleem, H.; Alessandro, A.D.; Downey, A.; Ceylan, H.; Materazzi, A.L. Dynamic characterization of a soft elastomeric capacitor for structural health monitoring. J. Struct. Eng. 2015, 141, 04014186. [CrossRef]

17. Sun, Q.J.; Zhuang, J.; Venkatesh, S.; Zhou, Y.; Han, S.T.; Wu, W.; Kong, K.W.; Li, W.J.; Chen, X.; Li, R.K.Y.; et al. Highly sensitive and ultrastable skin sensors for biopressure and bioforce measurements based on hierarchical microstructures. ACS Appl. Mater. Interfaces 2018, 10, 4086-4094. [CrossRef] [PubMed]

18. Shin, S.H.; Ji, S.; Choi, S.; Pyo, K.H.; An, B.W.; Park, J.; Kim, J.; Kim, J.Y.; Lee, K.S.; Kwon, S.Y.; et al. Integrated arrays of air-dielectric graphene transistors as transparent active-matrix pressure sensors for wide pressure ranges. Nat. Commun. 2017, 8, 14950. [CrossRef]

19. Choi, W.; Lee, J.; Yoo, Y.K.; Kang, S.; Kim, J.; Lee, J.H. Enhanced sensitivity of piezoelectric pressure sensor with microstructured polydimethylsiloxane layer. Appl. Phys. Lett. 2014, 104, 123701. [CrossRef]

20. Baek, S.; Jang, H.; Kim, S.Y.; Jeong, H.; Han, S.; Jang, Y.; Kim, D.H.; Lee, H.S. Flexible piezocapacitive sensors based on wrinkled microstructures: Toward low-cost fabrication of pressure sensors over large areas. RSC Adv. 2017, 7, 39420. [CrossRef]

21. Kim, Y.; Jang, S.; Kang, B.J.; Oh, H.J. Fabrication of highly sensitive capacitive pressure sensors with electrospun polymer nanofibers. Appl. Phys. Lett. 2017, 111, 073502. [CrossRef] 
22. Cao, H.; Thakar, S.K.; Oseng, M.L.; Nguyen, C.M.; Jebali, C.; Kouk, A.B. Development and characterization of a novel interdigitated capacitive strain sensor for structural health monitoring. IEEE Sens. J. 2015, 15, 6542-6548. [CrossRef]

23. Mannsfeld, S.C.; Tee, B.C.; Stoltenberg, R.M.; Chen, C.V.; Barman, S.; Muir, B.V.; Sokolov, A.N.; Reese, C.; Bao, Z. Highly sensitive flexible pressure sensors with microstructured rubber dielectric layers. Nat. Mater. 2010, 9, 859-864. [CrossRef] [PubMed]

24. Mohiuddin, M.; Van Hoa, S. Electrical resistance of CNT-PEEK composites under compression at different temperatures. Nanoscale Res. Lett. 2011, 6, 419. [CrossRef] [PubMed]

25. Xie, Y.; Yang, Q. Tyre-pavement contact stress distribution considering tyre types. Road Mater. Pavement. 2018, 19. [CrossRef]

26. Luo, R.; Prozzi, J.A. Strain distribution in the asphalt layer under measured 3-D Tire-pavement contact stresses. Road Mater. Pavement 2007, 8, 61-86. [CrossRef]

27. Quan, Y.; Wei, X.; Xiao, L.; Wu, T.; Pang, H.; Liu, T.; Huang, W.; Wu, S.; Chen, Z. Highly sensitive and stable flexible pressure sensors with micro-structured electrodes. J. Alloys Compd. 2017, 699, 824-831. [CrossRef]

28. Paredes-Madrid, L.; Fonseca, J.; Matute, A.; Gutiérrez Velásquez, E.; Palacio, C. Self-Compensated Driving Circuit for Reducing Drift and Hysteresis in Force Sensing Resistors. Electronics 2018, 7, 146. [CrossRef]

29. Kwon, M.T. Signal processing of piezoelectric weight-in-motion systems. In Proceedings of the Fifth IASTED International Conference on Circuits, Signals and Systems, Banff, AB, Canada, 2-4 July 2007; Pearson: London, UK, 1996; pp. 233-238, ISBN 978-0-88986-670-6.

30. Huang, Y.; Wang, L.; Hou, Y.; Zhang, W.; Zhang, Y. A prototype IOT based wireless sensor network for traffic information monitoring. Int. J. Pavement Res. Technol. 2018, 11, 146-152. [CrossRef]

(C) 2019 by the authors. Licensee MDPI, Basel, Switzerland. This article is an open access article distributed under the terms and conditions of the Creative Commons Attribution (CC BY) license (http://creativecommons.org/licenses/by/4.0/). 